\title{
Efeito do ácido esteárico em scaffolds de alumina obtidos por manufatura aditiva usando o método de fabricação por filamento fundido
}

\section{(Effect of stearic acid on alumina scaffolds obtained by additive manufacturing using the fused filament fabrication method)}

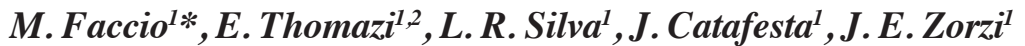 \\ ${ }^{1}$ Universidade de Caxias do Sul, R. Francisco Getúlio Vargas 1130, 95070-560, Caxias do Sul, RS, Brasil \\ ${ }^{2}$ Instituto Federal do Rio Grande do Sul, R. Avelino Antônio de Souza 1730, 95043-700, Caxias do Sul, RS, Brasil
}

\begin{abstract}
Resumo
As cerâmicas porosas podem ser utilizadas nas mais diversas aplicações, como na indústria petroquímica e na área da saúde. No entanto, é difícil produzir cerâmicas com formas complexas usando processos convencionais. Neste trabalho, diferentes termoplásticos (PEBD, ABS e PLA) foram utilizados para a extrusão de filamentos com alumina submicrométrica. Estes filamentos foram extrudados com e sem ácido esteárico que, posteriormente, foram utilizados na manufatura aditiva para a impressão de estruturas porosas (scaffolds) pelo método de fabricação por filamento fundido. Foram obtidas imagens por microscopia eletrônica de varredura das amostras com e sem o ácido esteárico, antes e após a sinterização. Estas imagens mostraram que a presença do ácido esteárico reduziu a formação de defeitos, pois além de atuar como dispersante também facilitou a posterior remoção do veículo orgânico do scaffold, antes da sinterização final.
\end{abstract}

Palavras-chave: fabricação por filamento fundido, alumina, ácido esteárico, microestrutura, biocerâmica.

Abstract

Porous ceramics can be used in the most diverse applications, such as in the petrochemical industry and in the health area. However, it is difficult to produce ceramics with complex shapes using conventional processes. In this work, different thermoplastics (LDPE, $A B S$, and PLA) were used for the extrusion of filaments with submicrometric alumina. These filaments were extruded with and without stearic acid and, subsequently, were used in additive manufacturing for printing porous structures (scaffolds) by fused filament fabrication. Scanning electron microscopy images of the samples with and without stearic acid were obtained before and after sintering. These images showed that the presence of stearic acid reduced the formation of defects since, in addition to acting as a dispersant, it also facilitated the subsequent removal of the organic vehicle from the scaffold, before final sintering.

Keywords: fused filament fabrication, alumina, stearic acid, microstructure, bioceramic.

\section{INTRODUÇÃO}

$\mathrm{Na}$ área da saúde e também na indústria petroquímica, as cerâmicas porosas são amplamente utilizadas pela sua capacidade de melhorar a eficiência catalítica, mimetizar a porosidade de sistemas naturais, além de fornecer área superficial adequada, entre outras vantagens. Neste caso, é necessária a produção de estruturas complexas com porosidade adequada, o que pode ser feito por impressão 3D, por ser um método rápido e que permite obter peças com elevada complexidade $[1,2]$. Atualmente o método utilizado para a obtenção destas estruturas é o da réplica (método de espuma), que permite um volume de poros abertos até $95 \%$, variando de nanômetros a micrômetros. Este método possui ainda aplicações na filtração de metais fundidos, filtros de exaustão de motores a diesel e filtração de gases quentes. A

*mfaccio1@ucs.br

๑https://orcid.org/0000-0002-0358-8941 principal desvantagem do método de espuma é a preparação da suspensão, onde as características reológicas devem apresentar uma fluidez ideal para a remoção, como também um comportamento pseudoplástico para revestir o molde (espuma) com eficiência. Devido a isso, a impressão 3D é uma alternativa de substituição para fabricação de peças de alta qualidade, com boas propriedades mecânicas, alta porosidade e estabilidade química em altas temperaturas $[1,3,4]$.

A fabricação por filamento fundido (FFF) ou FDM (fused deposition modeling) é um método popular de manufatura aditiva (AM), por ser considerado um processo mais simples, de baixo custo e que pode fazer uso de uma grande variedade de materiais. No método de FFF, um filamento termoplástico é puxado, aquecido e extrudado através de um bocal, com o auxílio de duas rodas de alimentação, sendo depositado sobre uma placa, que pode ou não ser aquecida, de baixo para cima, uma camada de cada vez $[5,6]$. A técnica de FFF também pode ser utilizada para a impressão de peças metálicas ou cerâmicas com formas complexas. As matérias-primas usadas consistem em um ligante/ 
veículo polimérico e uma alta carga de pó sinterizável [79]. Para a otimização do projeto de peças cerâmicas por AM, faz-se necessária a seleção do pó com propriedades adequadas, a utilização de ligantes para o veículo orgânico, que são responsáveis pelo transporte das partículas do pó cerâmico, um tratamento subsequente para a remoção do veículo orgânico (debinding) e a sinterização, que permitem a remoção dos ligantes, o coalescimento das partículas para aumento da densificação e a obtenção da peça final [10]. A deposição fundida de cerâmica (FDC) foi introduzida na década de 1990, com o uso de polímeros preenchidos com pó de cerâmica para a fabricação aditiva de peças cerâmicas. Embora os polímeros acrilonitrila butadieno estireno (ABS) e ácido polilático (PLA) sejam os filamentos mais comuns, o uso de precursores cerâmicos para a impressão 3D por FFF ainda é um desafio $[9,11,12]$. Outro polímero que poderia ser utilizado é o polietileno de baixa densidade (PEBD), que é caracterizado por apresentar propriedades particulares de grande interesse devido à ampla gama de aplicações e fácil processabilidade, além do baixo custo, que o torna uma alternativa de substituição para materiais com custo elevado no processo de manufatura aditiva $[13,14]$. Um dos pontos cruciais que determinam o sucesso do processo são as propriedades do filamento. O filamento deve possuir propriedades mecânicas adequadas para a impressora usada na FFF. Para a obtenção de um componente cerâmico denso e resistente por FFF/FDM, os filamentos termoplásticos devem ser preenchidos com teores de pós cerâmicos (sólidos) relativamente elevados, pois o alto nível de enchimento é necessário para garantir a sinterização adequada da cerâmica na etapa final do processamento $[8,9]$. Mas o elevado volume de sólidos dificulta a fabricação do filamento.

O processo FFF para impressão de uma peça cerâmica é mais complexo do que para a impressão de um termoplástico puro ou um polímero com um baixo teor de carga (normalmente $<20 \%$ em volume de carga inorgânica). Alguns parâmetros são fatores fundamentais, pois afetam a qualidade do produto final, tais como a concentração, tipo de material, viscosidade, tamanho médio das partículas do pó, diferença de fusão e adesão de cada material, morfologia, rugosidade da superfície e molhabilidade entre o pó e os ligantes poliméricos [15]. Devem ser levadas em consideração as vantagens e limitações do processo FFF. Custo, simplicidade de processo, velocidade de impressão e possibilidade de deposição de diversos materiais simultaneamente são os fatores mais benéficos, enquanto os parâmetros de otimização que interferem diretamente a construção da peça, deformações nas camadas e baixa qualidade superficial são consideradas limitações importantes. Por este motivo, o desenvolvimento de compósitos usando FFF permite a melhora das propriedades das peças impressas, mas ainda apresenta como desafio a formação de vazios e a interação (ligação) entre as fibras e a matriz e entre as cargas e a matriz [16-18]. O filamento obtido deve ser resistente suficiente para evitar cisalhamento devido à pressão da roda motriz e, também, rígido o suficiente para evitar empenamento entre as rodas motrizes e a zona quente da matriz de extrusão (bocal). Também deve ser notado que o comportamento de fusão do material, bem como os parâmetros de processamento, tem uma influência significativa no sucesso da impressão [8]. Uma baixa viscosidade é necessária para reduzir a resistência ao fluxo, e uma boa adesão é necessária entre as camadas já extrudadas e depositadas, bem como à plataforma de construção. De modo a obter todas essas propriedades em uma matériaprima, são necessários sistemas ligantes multicomponentes e com as proporções corretas de cada componente [7, 19].

O componente termoplástico e outros aditivos de processamento, como dispersantes/surfactantes, que são essenciais para o processo de impressão, devem ser completamente removidos antes da etapa de sinterização. Portanto, uma etapa de eliminação (debinding) é necessária [8]. A etapa de debinding é um processo fundamental para obtenção de peças cerâmicas íntegras, uma vez que o componente de menor temperatura de fusão é removido nos estágios iniciais e o restante dos ligantes poliméricos são eliminados durante a pré-sinterização em temperaturas mais elevadas $[8,9]$. $\mathrm{O}$ ácido esteárico é um destes aditivos e se destaca por promover a redução de aglomerações, além de homogeneizar a microestrutura, auxiliando na dispersão das partículas de pó no veículo orgânico conferindo estabilidade eletrostática, relacionada com a força de repulsão entre as partículas, pois quando as forças atrativas dominam o sistema, as partículas aglomeram e a viscosidade aumenta significativamente, interferindo no processo de impressão [20]. A etapa de extração do veículo orgânico é identificada como uma das principais causas de defeitos e, neste caso, o tamanho das partículas do pó e sua distribuição no filamento são fundamentais para permitir uma separação suave do veículo orgânico das partículas. Um ligante ideal se caracteriza por apresentar uma boa fluidez no processo de impressão, boa interação com a partícula e facilidade na etapa de remoção do mesmo. O ligante deve apresentar eficiência suficiente para decompor e evaporar sem danificar a estabilidade estrutural da amostra, mantendo uma resistência para etapa posterior de sinterização $[15,21$, 22]. A etapa de sinterização se caracteriza pelo processo no qual os agregados de pó, compactados ou não, são transformados em corpos sólidos por mecanismos de transporte atômico por difusão, pelo efeito do aquecimento em alta temperatura; ocorre a consolidação térmica das peças a verde, após a remoção dos aditivos poliméricos, pela redução da porosidade e da energia livre do sistema, pelo coalescimento das partículas, aumentando a resistência mecânica. É necessário o controle rigoroso de variáveis que podem afetar diretamente a formação da estrutura da peça como temperatura, tempo, atmosfera do forno, tamanho do pó e natureza do pó (fator porosidade) [23-25].

Neste trabalho foram utilizados diferentes materiais termoplásticos (ABS, PLA e PEBD) para obter um filamento com pó de alumina submicrométrica. Os filamentos foram extrudados com auxílio de ácido esteárico, para posterior impressão por fabricação por filamento fundido (FFF). O objetivo é produzir uma estrutura porosa complexa de 
alumina sinterizada (scaffold) para diferentes aplicações.

\section{MATERIAIS E MÉTODOS}

Foi utilizada uma alumina $\left(\mathrm{Al}_{2} \mathrm{O}_{3}\right)$ submicrométrica (A-1000SG, Almatis, EUA), como recebida; a composição e as propriedades, de acordo com o fabricante, foram: $99,8 \% \mathrm{Al}_{2} \mathrm{O}_{3}, 0,04 \% \mathrm{MgO}, 0,03 \% \quad \mathrm{SiO}_{2}, 0,02 \% \mathrm{Fe}_{2} \mathrm{O}_{3}$, $0,07 \% \mathrm{Na}_{2} \mathrm{O}$ e $0,02 \% \mathrm{CaO}$; área superficial de $8,4 \mathrm{~m}^{2} \cdot \mathrm{g}^{-1}$; densidade sinterizada $\left(\right.$ a $\left.1540{ }^{\circ} \mathrm{C}\right)$ de $3,85 \mathrm{~g} . \mathrm{cm}^{-3}$; retração na sinterização de $17 \%$; e tamanho médio de partícula de $0,4 \mu \mathrm{m}$. Para obtenção dos filamentos com pó de $\mathrm{Al}_{2} \mathrm{O}_{3}$, foram utilizados três polímeros termoplásticos diferentes: 1) polietileno de baixa densidade (LDPE, Roto-K Natura), pó com densidade de $0,937 \mathrm{~g} \cdot \mathrm{cm}^{-3}$ e temperatura de operação entre 180 e $220^{\circ} \mathrm{C} ; 2$ ) ácido polilático (PLA, GTMax3D), natural (transparente), pellets com densidade de $1,07 \mathrm{~g} . \mathrm{cm}^{-3}$, temperatura de operação entre 190 e $220^{\circ} \mathrm{C}$ e temperatura de transição vítrea de $60^{\circ} \mathrm{C}$; e 3 ) acrilonitrila butadieno estireno (ABS, Terluran GP-35 natural, INEOS Styrolution), pellets com densidade de $1,04 \mathrm{~g} . \mathrm{cm}^{-3}$, temperatura de operação entre 220 e $260{ }^{\circ} \mathrm{C}$ e temperatura de transição vítrea de $105^{\circ} \mathrm{C}$. $\mathrm{O}$ aditivo utilizado no processo de obtenção dos filamentos foi: ácido esteárico (AE) comercial (Simoquímica), pó com densidade de 0,85 a $0,9 \mathrm{~g} \cdot \mathrm{cm}^{-3}$ e temperatura de fusão entre 58 e $67^{\circ} \mathrm{C}$. As quantidades de cada componente usado para fazer o filamento encontram-se na Tabela I.

Tabela I - Composição (\% em massa) dos diferentes filamentos.

[Table I - Composition (wt\%) of the different filaments.]

\begin{tabular}{cccc}
\hline \multicolumn{2}{c}{ Polímero (\%) } & Alumina (\%) & AE (\%) \\
\hline LDPE & 45,5 & 45,5 & 9,0 \\
LDPE & 50,0 & 50,0 & - \\
ABS & 45,5 & 45,5 & 9,0 \\
ABS & 50,0 & 50,0 & - \\
PLA & 45,5 & 45,5 & 9,0 \\
PLA & 50,0 & 50,0 & - \\
\hline
\end{tabular}

A mistura de alumina e ligantes orgânicos (feedstock), para produção do filamento para FFF, foi preparada diretamente em uma extrusora dupla rosca (ES 35 FR, SEIBT, Brasil) com composições apresentadas na Tabela I. A mistura alumina/ligantes foi extrudada entre 170 e $200{ }^{\circ} \mathrm{C}$, velocidade de rosca de $200 \mathrm{rpm}$ e capacidade de processamento de $3 \mathrm{~kg} / \mathrm{h}$. Os materiais de partida foram alimentados na extrusora de dupla rosca com aquecedor multizona. A temperatura de trabalho ficou entre 180 e $200{ }^{\circ} \mathrm{C}$ no primeiro estágio. A matriz de extrusão com um diâmetro circular de $3 \mathrm{~mm}$ foi mantida a $190{ }^{\circ} \mathrm{C}$ para o ABS, $160{ }^{\circ} \mathrm{C}$ para o LDPE e $180{ }^{\circ} \mathrm{C}$ para o PLA. O filamento quente foi resfriado em uma calha com água e enrolado em um carretel, de modo a obter um filamento com diâmetro médio de 1,75 $\mathrm{mm}$ (diâmetro do bocal da extrusora da impressora 3D).
As medições de viscosidade foram realizadas no material fundido usando um reômetro capilar (Ceast SR20, Instron, EUA), com força máxima de $20 \mathrm{kN}$ e um furo padrão de $15 \mathrm{~mm}$ de diâmetro. As amostras foram estudadas nas temperaturas de: PEBD $190{ }^{\circ} \mathrm{C}$, ABS $220^{\circ} \mathrm{C}$ e PLA 160 ${ }^{\circ} \mathrm{C}$. Foi utilizada a termogravimetria para monitorar o comportamento térmico dos filamentos, tanto na impressão, como na extração dos ligantes. A termogravimetria (TG/ DTG) foi realizada em um analisador térmico (TGA 50, Shimadzu, Japão). Com base nos resultados das análises de TG, foram programadas as rampas de debinding e présinterização, em ar estático, usando um forno elétrico de laboratório (Sanchis).

A obtenção da estrutura aberta do scaffold foi feita em uma impressora 3D montada no próprio laboratório (impressora 3D de mesa e de código aberto, RepRap Graber i3), controlada por Arduino [26], junto ao módulo eletrônico Ramps 1.4. Para a impressão dos filamentos de ABS e PLA, foi utilizada velocidade de $50 \mathrm{~mm} / \mathrm{s}$. Para a impressão do PEBD foi utilizada a velocidade de $40 \mathrm{~mm} / \mathrm{s}$ (foi necessário diminuir a velocidade em função da pouca rigidez do filamento). $\mathrm{O}$ modelo computacional foi produzido utilizando o software SolidWorks e após salvo em arquivo STL compatível com a programação da impressora 3D. A geometria tridimensional do scaffold foi construída nas orientações de camadas de $0^{\circ}$ e $45^{\circ}$, com dimensão cúbica e diâmetro de filamento apresentada na Tabela II, de acordo com estudos já realizados para aplicação de scaffolds, de modo a evitar a formação de defeitos de empenamento das peças impressas [27]. Os scaffolds desenvolvidos pelo modelo computacional foram impressos com temperaturas de processamento listadas na Tabela III e com as dimensões cúbicas apresentadas na Tabela II.

Após a impressão, as peças cerâmicas a verde foram submetidas ao debinding imersas em leito de pó de alumina (A-1000SG) até a temperatura de $450^{\circ} \mathrm{C}$. A etapa de remoção do veículo orgânico para as misturas alumina/polímero iniciou em temperatura ambiente até $185^{\circ} \mathrm{C} \mathrm{com} \mathrm{taxa} \mathrm{de} 1{ }^{\circ} \mathrm{C} / \mathrm{min}$, permanecendo $10 \mathrm{~h}$ nesta temperatura; após, aquecimento até 410,385 ou $290{ }^{\circ} \mathrm{C}$ para as misturas com PEBD, ABS ou PLA com taxa de $1^{\circ} \mathrm{C} /$ min permanecendo por $10 \mathrm{~h}$ nesta temperatura e, por fim, aquecimento até $450{ }^{\circ} \mathrm{C}$ com taxa de $1{ }^{\circ} \mathrm{C} / \mathrm{min}$ permanecendo por $2 \mathrm{~h}$ nesta temperatura. Para a etapa de remoção, o tempo de permanência para todas as misturas foi de $29 \mathrm{~h}$ até $450{ }^{\circ} \mathrm{C}$. Em seguida, as peças foram retiradas do leito e pré-sinterizadas ao ar a $1000^{\circ} \mathrm{C} / 1$ $\mathrm{h}$ em um forno elétrico (Sanchis) para que o restante dos aditivos fossem removidos; para a sinterização também ao ar em forno elétrico (Blue M BF51634PCOMC, Lindberg, EUA), as peças foram colocadas sobre uma superfície refratária recoberta com uma fina camada de pó de alumina (A-1, Alcoa), com rampa de aquecimento de $1{ }^{\circ} \mathrm{C} / \mathrm{min}$ até $1600{ }^{\circ} \mathrm{C}$. A sinterização foi processada por mais $2 \mathrm{~h}$ nesta temperatura $[28,29]$.

As informações microestruturais foram obtidas por microscopia eletrônica de varredura (MEV), em um microscópio eletrônico de emissão de campo (FEG, 
Tabela II - Dimensões dos scaffolds (com e sem ácido esteárico).

[Table II - Dimensions of the scaffolds (with and without stearic acid).]

\begin{tabular}{cccccc}
\hline Feedstock & $\begin{array}{c}\text { Altura } \\
(\mathrm{mm})\end{array}$ & $\begin{array}{c}\text { Lado 1 } \\
(\mathrm{mm})\end{array}$ & $\begin{array}{c}\text { Lado 2 } \\
(\mathrm{mm})\end{array}$ & $\begin{array}{c}\text { Espaçamento entre } \\
\text { filamentos }(\mathrm{mm})\end{array}$ & $\begin{array}{c}\text { Diâmetro do fio } \\
\text { impresso }(\mathrm{mm})\end{array}$ \\
\hline Alumina/PEBD & 10 & 30 & 30 & 0,300 & 1 \\
Alumina/ABS & 10 & 30 & 30 & 0,300 & 1 \\
Alumina/PLA & 10 & 30 & 30 & 0,300 & 1 \\
\hline
\end{tabular}

Tabela III - Temperaturas de processamento para impressão 3D. [Table III - Processing temperatures for 3D printing.]

\begin{tabular}{ccc}
\hline Feedstock & $\begin{array}{r}\text { Temperatura do } \\
\text { bico extrusor }\left({ }^{\circ} \mathrm{C}\right)\end{array}$ & $\begin{array}{r}\text { Temperatura } \\
\text { da mesa }\left({ }^{\circ} \mathrm{C}\right)\end{array}$ \\
\hline Alumina/PEBD* & 220 & 40 \\
Alumina/ABS* & 250 & 60 \\
Alumina/PLA* & 190 & 40 \\
\hline
\end{tabular}

MIRA3, Tescan, Rep. Tcheca) operado com tensão de aceleração de $10 \mathrm{kV}$. A microscopia foi usada para observar a morfologia e defeitos microestruturais. A distribuição de tamanho de poros após sinterização, nas imagens de microscopia (ampliação de 50x), foi analisada usando o software ImageJ. As micrografias foram obtidas no modo de elétrons retroespalhados (BSE) para destacar o contraste das partículas de alumina imersas no polímero. A seção transversal das amostras foi obtida através de fratura criogênica em nitrogênio líquido e as demais micrografias foram obtidas com a fixação das amostras em portaamostras, ambas após a deposição de uma camada de Au por magnetron sputtering. Por fim, foram obtidas imagens das amostras sinterizadas.

\section{RESULTADOS E DISCUSSÃO}

Viscosidade: as medidas de viscosidade dos filamentos em função da taxa de cisalhamento estão apresentadas na Fig. 1. O objetivo deste ensaio foi verificar o efeito da presença ou não do ácido esteárico nas misturas dos polímeros com alumina. Todos os ensaios apresentaram um comportamento pseudoplástico, com uma redução significativa da viscosidade dos materiais processados com a presença do ácido esteárico, e a viscosidade diminuiu em todas as taxas de cisalhamento [8]. O comportamento pseudoplástico é essencial para garantir a estrutura da peça; além disso, foi possível observar que a adição de ácido esteárico provocou uma diminuição da viscosidade, o que facilitou o processo de impressão das peças com alumina. A introdução de aditivos como o ácido esteárico em misturas com pós cerâmicos reduz a aglomeração do sistema e homogeneíza a microestrutura conferindo estabilidade estérica, relacionada com a força de repulsão entre as partículas $[20,30]$. A redução exponencial nos três gráficos dos filamentos com PLA, ABS e PEBD condiz no fato de que o ácido esteárico atuou também como um

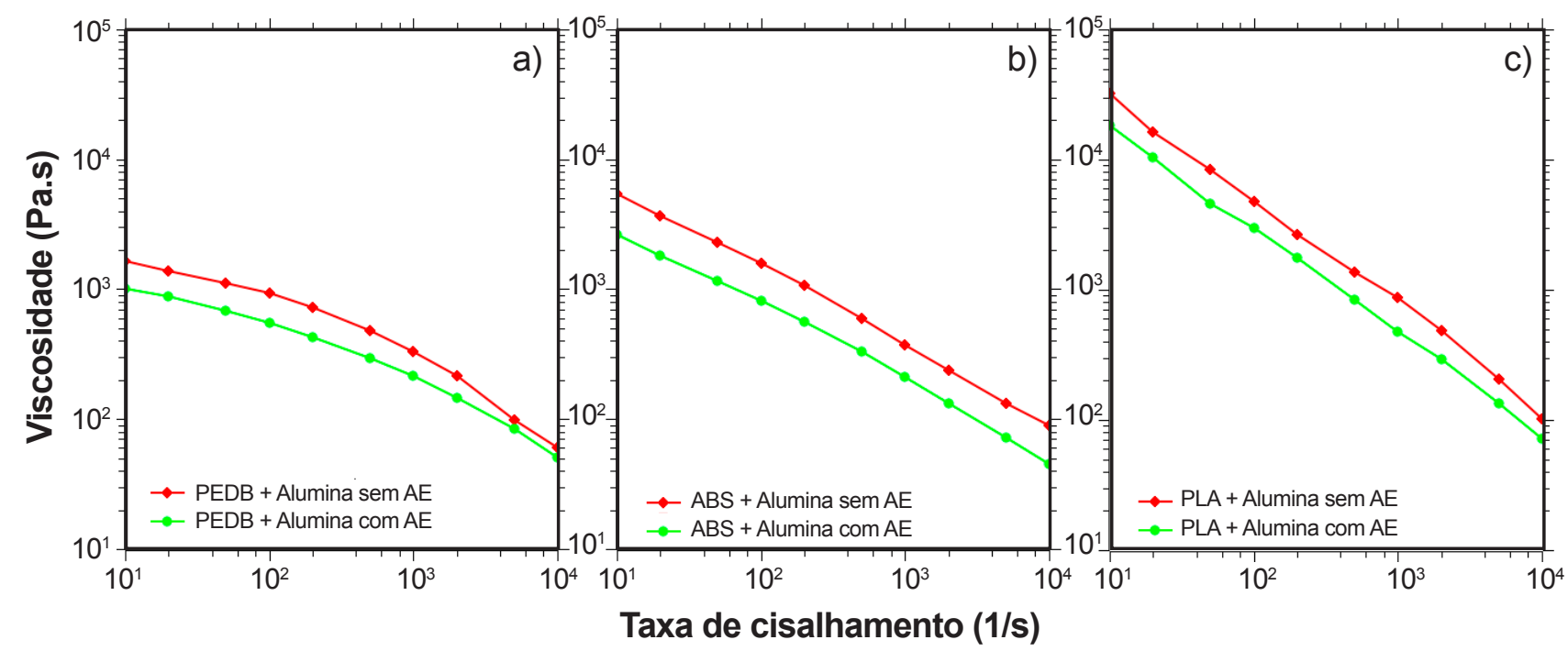

Figura 1: Viscosidade em função da taxa de cisalhamento para os filamentos produzidos com diferentes polímeros: a) PEBD (190 $\left.{ }^{\circ} \mathrm{C}\right)$; b) $\operatorname{ABS}\left(220^{\circ} \mathrm{C}\right)$; e c) PLA $\left(160^{\circ} \mathrm{C}\right)$.

[Figure 1: Viscosity as a function of shear rate for filaments produced with different polymers: a) LDPE (190 $\left.\left.{ }^{\circ} \mathrm{C}\right) ; b\right) \mathrm{ABS}\left(220^{\circ} \mathrm{C}\right) ;$ and c) PLA $\left.\left(160^{\circ} \mathrm{C}\right).\right]$ 
lubrificante interno da mistura, facilitando a mobilidade das partículas, ficando claro que sua ausência aumentou os valores de viscosidade. Uma viscosidade ideal e não elevada das misturas permite a fluidez do material pelo bico de impressão e um total preenchimento das camadas impressas [31].

Termogravimetria: a decomposição térmica de todas as misturas foi bastante semelhante, como pode ser observado na Fig. 2, com uma perda de massa gradual e temperatura de degradação $\left(\mathrm{T}_{\mathrm{d}}\right.$ ) entre 343 e $425^{\circ} \mathrm{C}$ para as amostras com ácido esteárico, sendo que os valores de $\mathrm{T}_{\mathrm{d}}$ para o PEBD foi de $424,38{ }^{\circ} \mathrm{C}$, para o ABS de $405,87^{\circ} \mathrm{C}$ e para o PLA de $326,94{ }^{\circ} \mathrm{C}$. A perda de massa perceptível em temperaturas mais baixas, de 185 a $300{ }^{\circ} \mathrm{C}$, é característica do ácido esteárico $[8,32]$. Nas amostras de PEBD, ABS e PLA sem ácido esteárico foi possível observar uma degradação típica destes materiais em todas as misturas, com o pico de DTG (derivada de TG) levemente deslocado para a direita (maior temperatura) em todas as amostras. O principal objetivo da obtenção das curvas de TG/DTG das misturas de cerâmicas com polímeros e ácido esteárico foi determinar a temperatura e o tempo de permanência nas etapas de debinding, de modo a evitar a formação de defeitos, como bolhas e rachaduras. Como o ácido esteárico possui menor temperatura de fusão, além de atuar como dispersante, ele é removido em temperatura menor, deixando canais abertos que são utilizados pelos polímeros para migrar para a superfície da peça $[25,33]$. Nas regiões observadas nas curvas de TG/DTG com as altas taxas de perda de massa descritas anteriormente $\left(\mathrm{T}_{\mathrm{d}}\right.$ e $\mathrm{T}_{\text {onset }}$ ), foi determinado um tempo maior de permanência no forno para que uma remoção gradual dos ligantes pudesse ser obtida.

Microscopia eletrônica de varredura dos filamentos: os filamentos de alumina/PEBD, alumina/ABS e alumina/ PLA (com e sem AE) obtidos por extrusão não apresentaram defeitos externos de processamento e nem falhas visíveis a olho nu, apresentando uma estrutura lisa e maleável que permitiu o bobinamento dos fios. Mesmo com a quantidade de material cerâmico adicionada na mistura de 45,5\% e $50 \%$ em massa, a extrusora não apresentou rejeição de processo, porém, pôde-se notar uma maior facilidade no controle dos parâmetros de processamento das misturas com adição do ácido esteárico, que atuou como dispersante no veículo orgânico, reduzindo o atrito entre as partículas do pó e as paredes da cavidade da extrusora durante o processamento [34]. A análise da seção transversal do fio possibilitou observar a presença de defeitos internos (Fig. 3). Evidenciados em estudos anteriores, a presença de poros internos está associada ao processo extrusão, devido ao fato de a condutividade térmica dos polímeros ser inferior à dos cerâmicos; neste caso, ela limita a taxa na qual o polímero pode ser aquecido e plastificado [35]. Assim, quando resfriado, essa baixa condutividade provoca encolhimento e não uniformidade, resultando em defeitos como delaminações, tensões de congelamento, deformações do extrudado e vazios, como pode ser observado na Fig. 3. No caso da mistura de PEBD sem AE, esse defeito pode
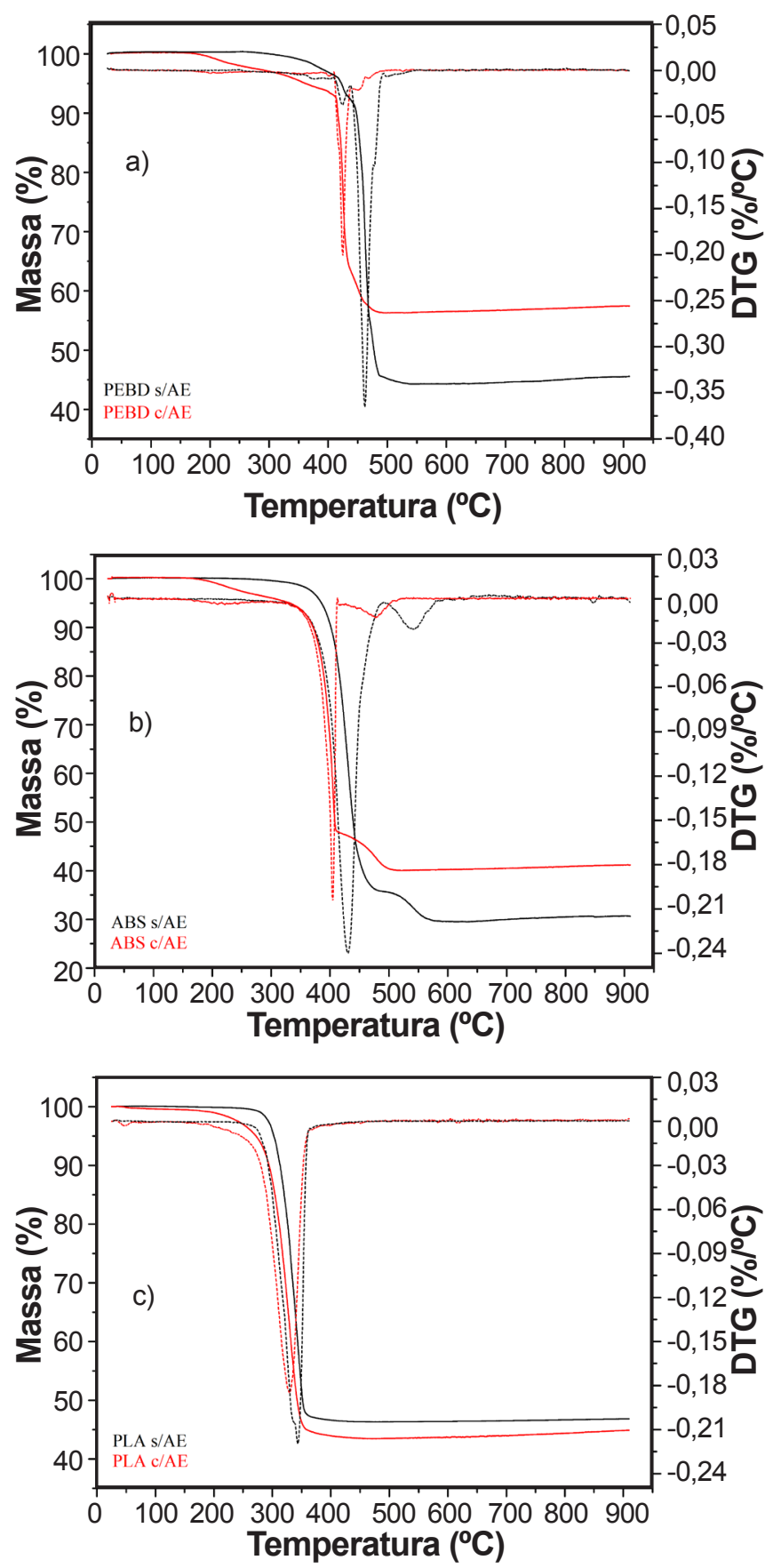

Figura 2: Curvas de análise termogravimétrica de alumina/PEBD, alumina/ABS e alumina/PLA (com e sem ácido esteárico).

[Figure 2: TG/DTG curves of alumina/LDPE, alumina/ABS, and alumina/PLA (with and without stearic acid).]

estar associado aos mais diversos fatores do processo de extrusão, como ventilação ineficiente, porta de ventilação obstruída, vácuo muito baixo, aprisionamento de ar, voláteis e até mesmo tamanho e formato da partícula. Também pode ser problema do resfriamento, quando o filamento passa pela canaleta com água após extrusão, não havendo tempo suficiente para boa uniformidade dos filamentos antes da solidificação, resultando em vazios [36]. Esse defeito não é prejudicial, já que o objetivo final é a obtenção do scaffold 

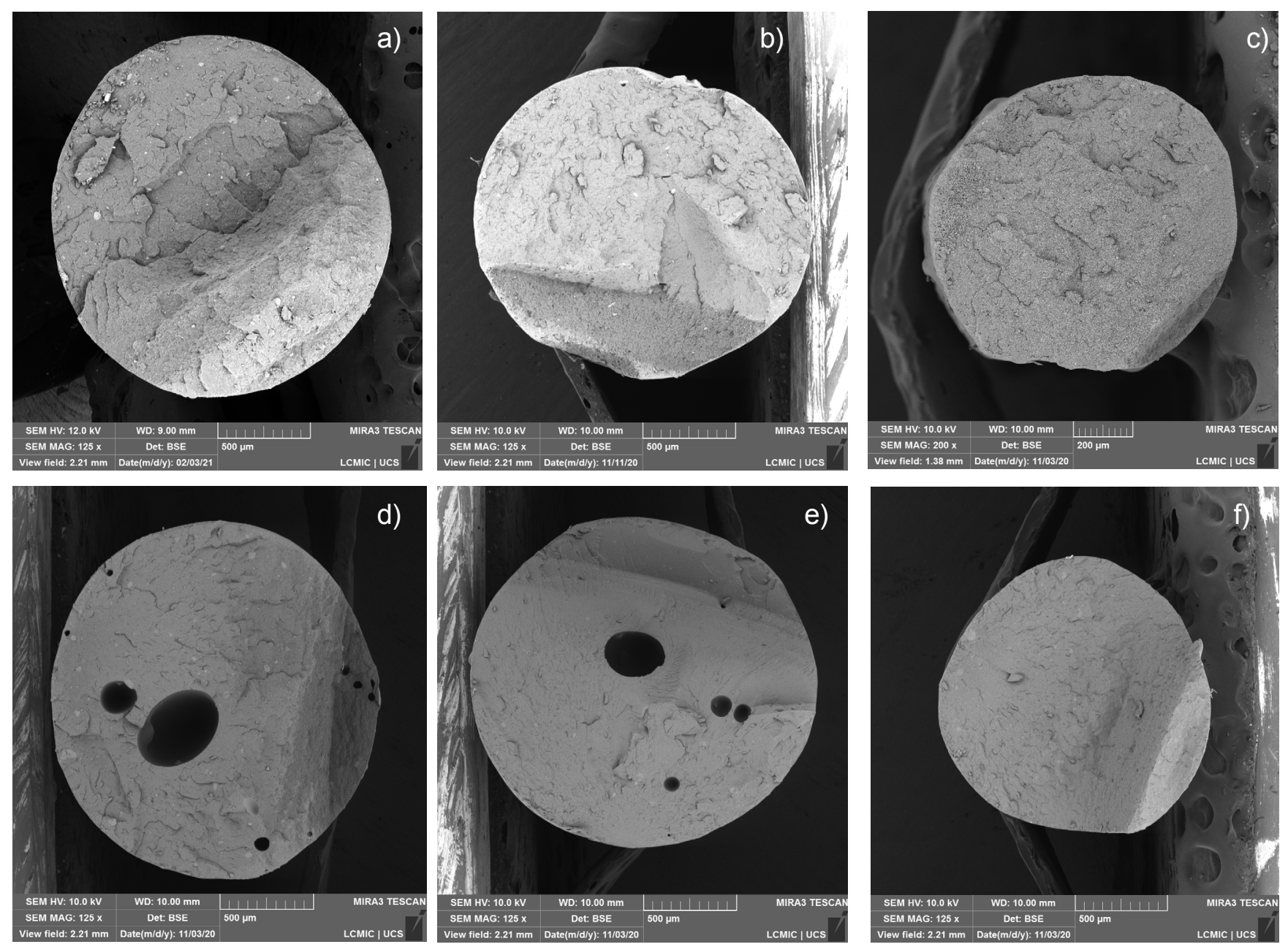

Figura 3: Imagens de MEV da seção transversal dos filamentos de alumina/PEBD (a,d), alumina/ABS (b,e) e alumina/PLA (c,f) obtidos por extrusão com $(\mathrm{a}, \mathrm{b}, \mathrm{c})$ e sem $(\mathrm{d}, \mathrm{e}, \mathrm{f})$ ácido esteárico.

[Figure 3: SEM images of cross-section of filaments of alumina/LDPE $(a, d)$, alumina/ABS $(b, e)$, and alumina/PLA $(c, f)$ obtained by extrusion with $(a, b, c)$ and without $(d, e, f)$ stearic acid.]

cerâmico, e esses vazios são eliminados durante as etapas posteriores, como a impressão, uma vez que o filamento passa novamente por uma nova extrusão, sendo eliminado totalmente nos processos de debinding e sinterização.

Por meio das imagens obtidas por microscopia, foi possível visualizar a distribuição das partículas, a interação do polímero com a cerâmica nas misturas, bem como a seção transversal das estruturas obtidas. A geometria, o tamanho e a distribuição das partículas podem afetar diretamente a qualidade da peça final. As partículas finas preenchem os espaços vazios, produzindo peças a verde de alta densidade; portanto, a quantidade de veículo orgânico também é um parâmetro chave para peças de alta densidade [37]. Pode-se perceber nas micrografias apresentadas que o ácido esteárico atuou como dispersante entre o polímero e partículas cerâmicas (Figs. 4a a 4c) [25, 28, 29]. A etapa mais importante na preparação de uma mistura é a dispersão do pó cerâmico no veículo orgânico para prevenir a aglomeração. Vários aditivos podem ser usados como agentes dispersantes em misturas que contenham polímeros e pó cerâmico, que é o caso do ácido esteárico.
Para proporcionar a adsorção química das moléculas na superfície das partículas, o dispersante deve possuir um grupo funcional adaptado às características do pó cerâmico que é utilizado. Como resultado da adsorção química do ácido esteárico na superfície da alumina, a viscosidade da mistura foi reduzida (Fig. 1), minimizando a segregação do ligante e do pó durante a moldagem e facilitando sua extração. Para óxidos cerâmicos como a alumina, a afinidade de adsorção à superfície do pó é fortemente dependente do tipo de ácido adicionado à mistura. $\mathrm{O}$ ácido esteárico reage com a superfície dos óxidos cerâmicos (mais especificamente com os grupos $\mathrm{OH}$ presentes na superfície das partículas do pó cerâmico) melhorando a molhabilidade entre o pó e os ligantes da mistura. O ácido esteárico faz isso reduzindo o ângulo de contato, pela diminuição da energia superficial na interface ligante-pó. Como consequência, sua adição facilita a mistura dos ligantes com o pó, obtendo-se melhor homogeneidade. Ele também atua como lubrificante nas paredes da matriz de extrusão [25, 38]. Para o processo FFF é necessária uma escolha adequada das matériasprimas, pois as características de tamanho, morfologia 

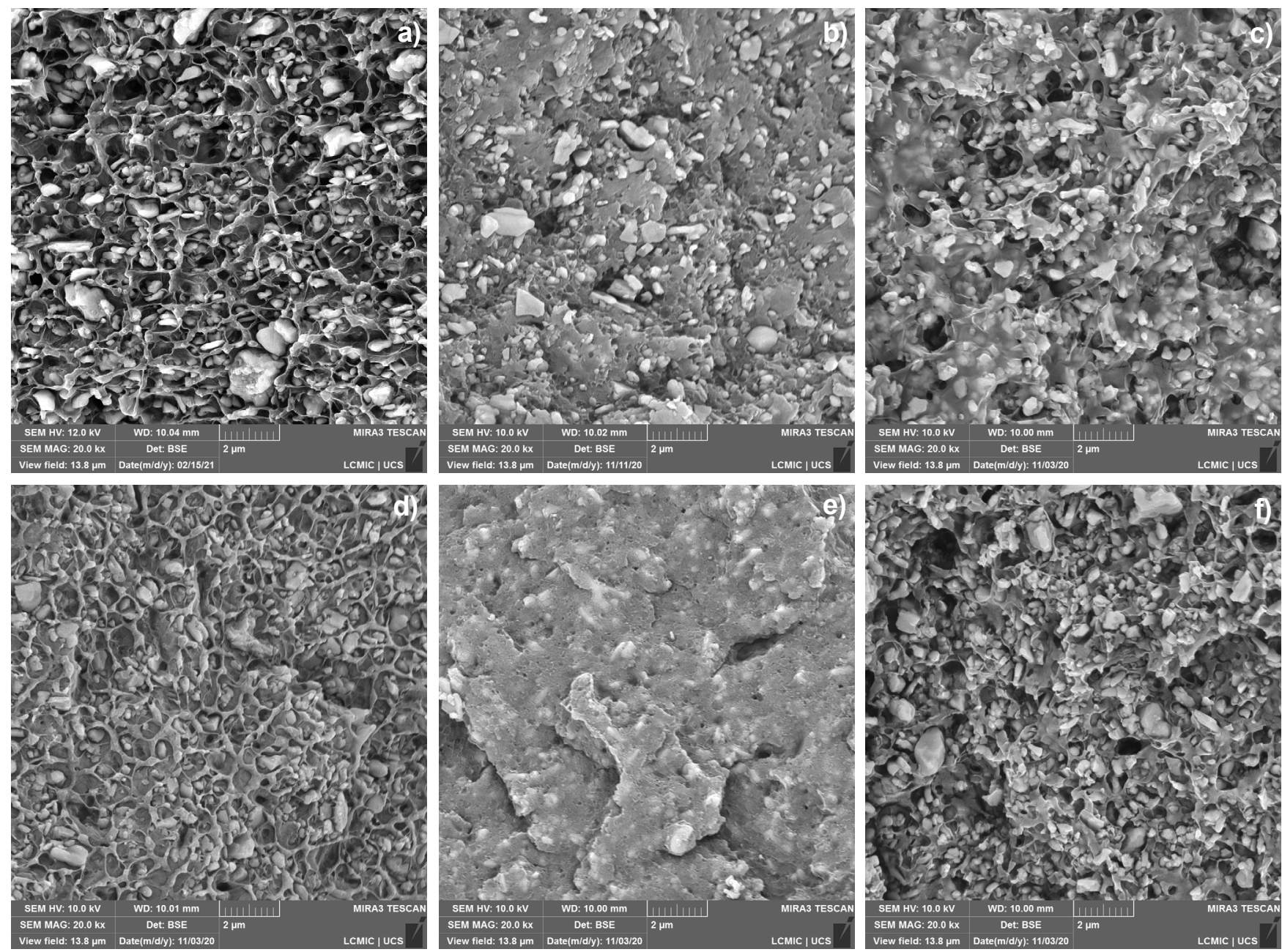

Figura 4: Micrografias de MEV de filamentos de PEBD (a,d), ABS (b,e) e PLA (c,f) com alumina, e com (a,b,c) e sem (d,e,f) ácido esteárico. [Figure 4: SEM micrographs of filaments of LDPE $(a, d), A B S(b, e)$, and PLA $(c, f)$ with alumina, and with $(a, b, c)$ and without $(d, e, f)$ stearic acid.]

e composição química do pó podem levar a alterações de propriedades impedindo muitas vezes a impressão, levando a uma interação distinta com o sistema ligante alterando fatores como rigidez e viscosidade [39].

Impressão 3D: a impressão de scaffolds de alumina com os polímeros PEBD, ABS e PLA, utilizados como veículos orgânicos, foi possível de ser realizada (Fig. 5), mas foi a presença do aditivo ácido esteárico que permitiu uma melhor conformação e precisão dimensional da peça acabada, além de conferir plasticidade à mistura, moldabilidade e transporte do pó cerâmico, melhorando a impressão da peça final. A alta quantidade de partículas cerâmicas dificultou a impressão, mas o uso do ácido esteárico como dispersante reduziu esta dificuldade e o efeito de expansão na extrusão tornou-se menos evidente em temperaturas mais elevadas, o que garantiu uma melhor precisão dimensional. Aditivos e surfactantes, como o ácido esteárico, atuam como lubrificantes durante a impressão, pois formam uma fina camada na superfície externa das partículas cerâmicas reduzindo o atrito e permitindo um empacotamento mais homogêneo.

No processo de impressão 3D foi possível observar diferenças no comportamento de cada material utilizado, cada qual com suas características e parâmetros de configuração de impressão. Na composição alumina/PEBD foi possível identificar que, devido às características do PEBD, ocorreu o resfriamento do filamento instantaneamente, provocando empenamento da estrutura após impressão, conforme pode ser visto na Fig. 6. Outro motivo possível associado ao defeito de empenamento é o fluxo de escoamento não tão contínuo. O PEBD é um polímero com maior taxa de encolhimento em volume e após a solidificação ocorreu alto grau de empenamento, não aderindo facilmente à plataforma de impressão. Este escoamento mais turbulento no momento da impressão foi devido à grande quantidade de material passando pelo bico de impressão, interferindo na precisão de impressão, como também na distribuição homogênea de temperatura, criando tensões residuais dentro da peça, pois ao depositar o filamento fundido camada por camada a energia térmica foi dissipada pela superfície superior, sendo que as camadas impressas anteriores já estavam resfriadas, provocando o encolhimento e gerando tensões de tração [4042].

Microscopia eletrônica de varredura dos scaffolds sinterizados: as amostras também foram analisadas por MEV, 

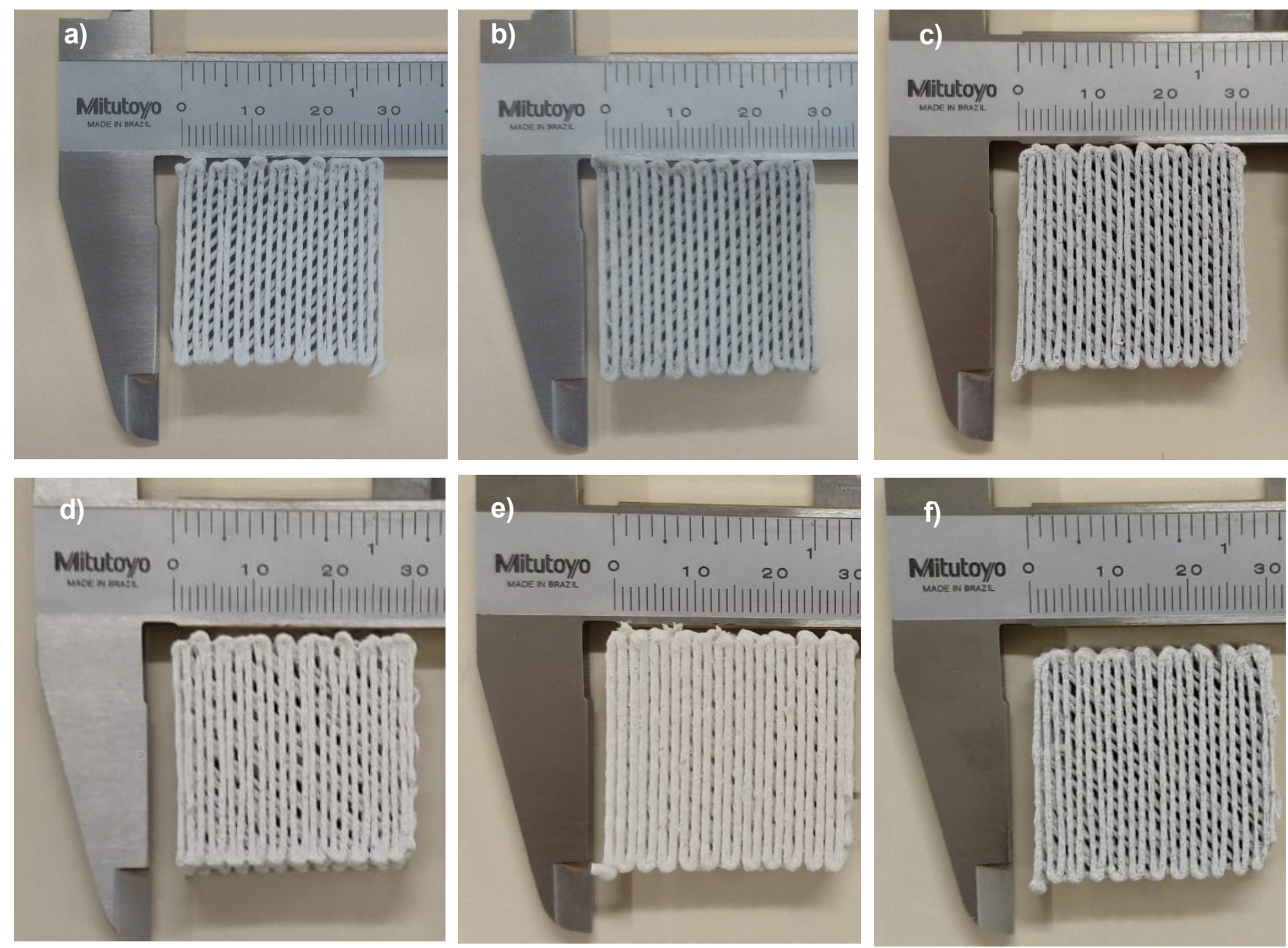

Figura 5: Imagens de scaffolds produzido por impressão 3D do PEBD (a,d), ABS (b,e) e PLA (c,f) com alumina, e com (a,b,c) e sem (d,e,f) ácido esteárico.

[Figure 5: Images of scaffolds produced by $3 D$ printing of $\operatorname{LDPE}(a, d), A B S(b, e)$, and PLA (c,f) with alumina, and with (a,b,c) and without $(d, e, f)$ stearic acid.]

após a sinterização. Como se observam nas macroestruturas da Fig. 7, os scaffolds apresentaram uma porosidade inerente em toda a estrutura, como também no filamento. Nas misturas de PEBD (com e sem AE), foi possível observar poros grandes nas imagens quando comparadas com as demais, mesmo em uma baixa ampliação, o que foi um indicativo de que este polímero apresentou características de processamento com maior taxa de encolhimento em volume, que resfriou rapidamente e apresentou alto grau de empenamento após solidificação, não aderindo facilmente à plataforma de impressão. Além disso, criaram-se tensões residuais dentro da peça, pois a energia térmica foi dissipada pela superfície superior, sendo que as camadas impressas anteriores já haviam resfriadas, provocando o encolhimento e gerando tensões de tração, sendo possível identificar a diferença de porosidade [40-42]. Além disso, ele não é utilizado normalmente no processo de impressão, o que se tornou um desafio para a produção dos scaffolds utilizando-o no veículo orgânico. Já nas demais misturas, foi possível observar uma distribuição de poros internos no filamento, um tamanho de poro desejado que confirmou uma boa impressão dos scaffolds, como também, um processo de debinding satisfatório (mantendo a resistência estrutural do scaffold), que permitiu uma eliminação do veículo orgânico e a sinterização completa com manutenção da estrutura cerâmica porosa.

Os tamanhos de poros presentes nos scaffolds produzidos estavam distribuídos nas mais diversas dimensões (Fig. 8), sendo no PEBD de 200 a $400 \mu \mathrm{m}$, para o ABS entre 110 e 200 $\mu \mathrm{m}$ e para o PLA foi entre 100 e $200 \mu \mathrm{m}$. Para aplicação de scaffold na área da saúde, uma porosidade adequada é aquela que promove o crescimento celular, como também na área industrial em catalisadores, que permite a dispersão de sítios ativos, meio filtrante, adsorvente e suporte catalítico para diversas reações químicas e tratamentos ambientais. Abbasi et al. [43] determinaram que, para uma melhor interação entre tecido ósseo, crescimento celular, vascularização e permeabilidade, com formação de osso maduro, uma faixa de trabalho aceitável de tamanho de poro é de 100 a $600 \mu \mathrm{m}$ para aplicação em tecido ósseo, compatibilizando a estrutura obtida neste estudo. Foi observado que o ácido esteárico foi fundamental para o processamento, pois além de permitir 

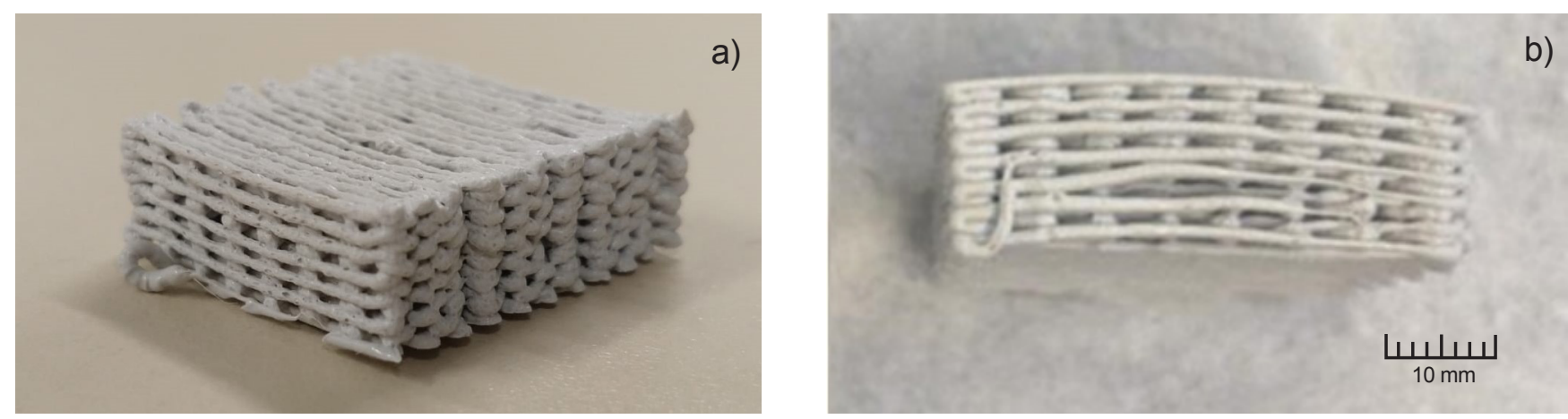

Figura 6: Imagens de scaffold de alumina/PEBD com AE mostrando defeito de empenamento.

[Figure 6: Images of alumina/LDPE scaffold with stearic acid showing distortion defect.]
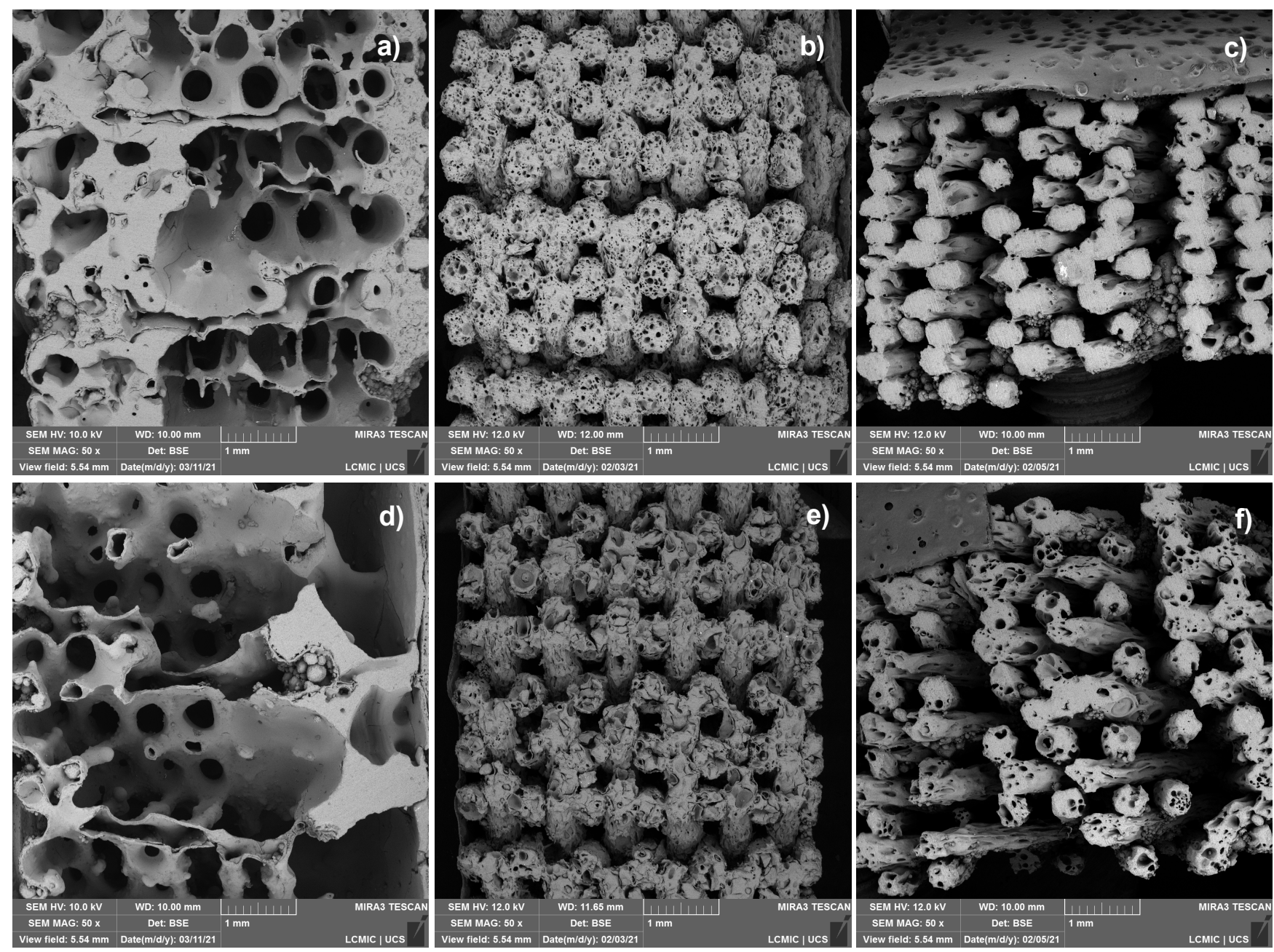

Figura 7: Imagens de MEV de baixo aumento das peças sinterizadas de alumina preparadas com filamentos contendo PEBD (a,d), ABS (b,e) e PLA (c,f), com (a,b,c) e sem (d,e,f) ácido esteárico.

[Figure 7: SEM images of low magnification of alumina sintered parts prepared with filaments containing LDPE (a,d), ABS (b,e), and PLA $(c, f)$, with $(a, b, c)$ and without $(d, e, f)$ stearic acid.]

uma facilidade de impressão, facilitou a etapa de debinding e sinterização, permitindo deixar os poros abertos, de modo que o veículo orgânico pudesse ser eliminado totalmente sem comprometer a estrutura observada no estudo desenvolvido para todos os scaffolds [22].

Nas imagens da Fig. 9 é possível identificar uma sinterização adequada para todas as misturas; o tamanho de partícula cresceu em uma ordem de magnitude em relação ao pó cerâmico. Também foi observado que em todas as misturas a alumina ficou bem sinterizada, formando um corpo cerâmico denso, o que indicou um bom processamento desde a produção dos filamentos, impressão dos scaffolds 

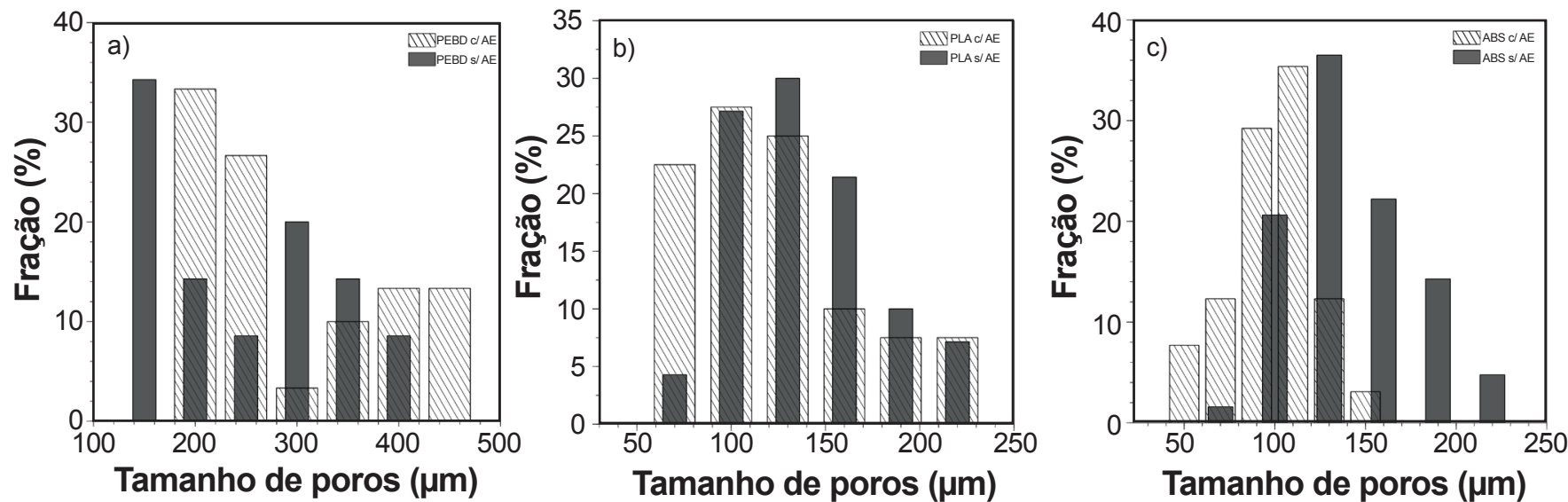

Figura 8: Distribuição dos tamanhos de poros nos scaffolds sinterizados preparados com e sem AE: a) alumina/PEBD; b) alumina/ABS; e c) alumina/PLA.

[Figure 8: Distribution of pore sizes in sintered scaffolds prepared with and without stearic acid: a) alumina/LDPE; $b$ ) alumina/ABS; and c) alumina/PLA.]
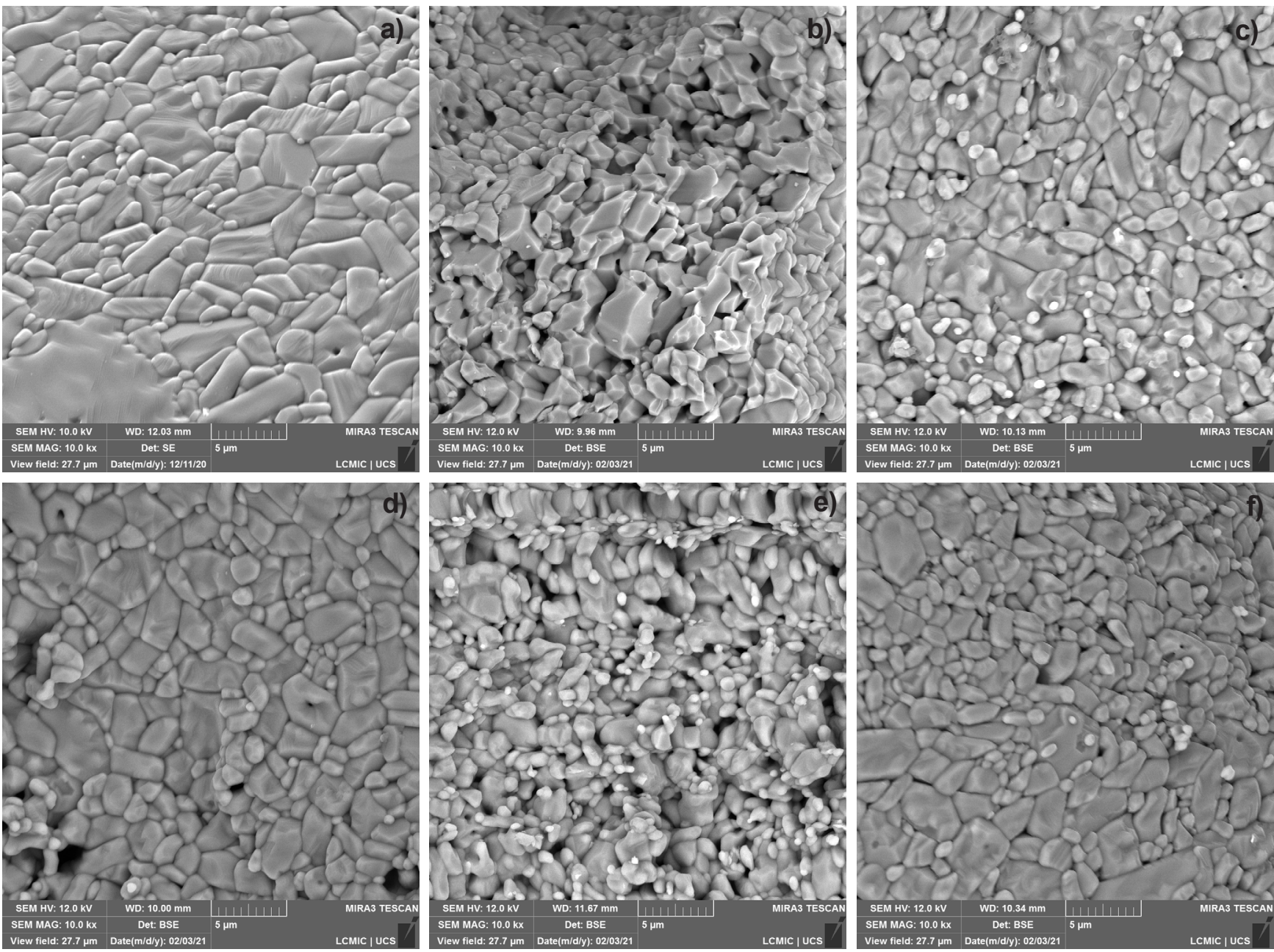

Figura 9: Micrografias de MEV dos scaffolds sinterizados que foram impressos com misturas contendo PEBD (a,d), ABS (b,e) e PLA (c,f), com $(\mathrm{a}, \mathrm{b}, \mathrm{c})$ e sem $(\mathrm{d}, \mathrm{e}, \mathrm{f})$ ácido esteárico.

[Figure 9: SEM micrographs of sintered scaffolds printed with mixtures containing $\operatorname{LDPE}(a, d), A B S(b, e)$, and $P L A(c, f)$, with $(a, b, c)$ and without $(d, e, f)$ stearic acid.]

e remoção do veículo orgânico (debinding). Finalmente,

a etapa de sinterização sempre pode ser otimizada de acordo com sua composição química, presença de veículos orgânicos, temperatura utilizada e tempo de permanência, 
viabilizando um processo de obtenção de peças cada vez mais eficiente, com propriedades adequadas para cada aplicação [44].

\section{CONCLUSÕES}

Foram preparados filamentos com três diferentes

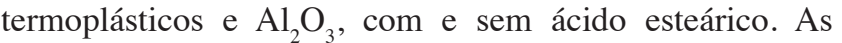
micrografias dos filamentos permitiram identificar que a presença de ácido esteárico auxiliou na dispersão das partículas cerâmicas nos polímeros. As medidas de reologia mostraram um comportamento pseudoplástico, com redução da viscosidade com adição do ácido esteárico, diminuindo a aglomeração do sistema e homogeneizando a mistura. Na etapa de impressão 3D das estruturas do tipo scaffold, a mistura alumina com PEBD apresentou pequenos defeitos no processo de impressão, que provocou o empenamento/ distorção da estrutura, não aderindo facilmente à plataforma de impressão. As demais misturas foram satisfatórias mesmo com uma alta quantidade de pó cerâmico. A presença do ácido esteárico facilitou o debinding, reduzindo ou evitando a formação de defeitos, como bolhas e rachaduras. A sinterização dos scaffolds obtidos com todas as misturas (PEBD, ABS e PLA) foi satisfatória, com características de compactação ideais, coalecimento total das partículas de alumina, boa interação entre as camadas depositadas e apresentando uma estrutura sólida mesmo com porosidade elevada, adequada para aplicação em tecido ósseo. O filamento produzido com a mistura alumina/ABS apresentou melhores propriedades de desempenho em todas as etapas, sem formação de defeitos, com facilidade de processo de impressão e pré-sinterização, com resistência mais elevada da estrutura e uma sinterização com uma microestrutura microporosa satisfatória. As misturas de alumina/PEBD foram as que apresentaram tamanhos de poros maiores, acima de $200 \mu \mathrm{m}$, o que em teoria permite a sua aplicação em crescimento ósseo. Por fim, concluiu-se que é possível produzir scaffolds cerâmicos por manufatura aditiva, utilizando impressão 3D de mistura cerâmico/polímero. É necessário modificar os parâmetros de processamento para cada polímero e etapa de manufatura, para finalmente obter uma peça cerâmica porosa de boa qualidade. A otimização de fatores como quantidade de aditivos, temperaturas e tempos permite a obtenção de peças com eficiência, com propriedades adequadas para cada aplicação. A porosidade observada na estrutura final variou conforme o polímero utilizado, o que permite optar pelo polímero mais adequado para aplicações específicas, como scaffolds com porosidade controlada para aplicação em biocerâmicas, catálise ou filtros.

\section{AGRADECIMENTOS}

Agradecemos o apoio do Conselho Nacional de Desenvolvimento Científico e Tecnológico (CNPq) bolsa 305528/2018-1, e da Universidade de Caxias do Sul (UCS). Este estudo também foi parcialmente financiado pela Coordenação de Aperfeiçoamento de Pessoal de Nível Superior - Brasil (CAPES) - Código 001 e Financiadora de Estudos e Projetos (FINEP). Os autores gostariam de agradecer ao Laboratório Central de Microscopia (LCMIC) e, em especial, a Rodrigo A. Barbieri pelo auxílio na obtenção das imagens de microscopia eletrônica de varredura. Agradecemos também aos técnicos do Laboratório de Polímeros da Universidade de Caxias do Sul (LPol), principalmente ao Baltazar Dutra e Damiani Paludo Budcke pelo processamento por extrusão e pelas medidas de análise térmica. Os autores também agradecem o Laboratório de Reologia do IFRS - Caxias do Sul pelas análises reológicas.

\section{REFERÊNCIAS}

[1] C. Huo, X. Tian, Y. Nan, D. Li, J. Eur. Ceram. Soc. 40 (2020) 12.

[2] A. Lind, O. Vistad, M.F. Sunding, K.A. Andreassen, J.H. Cavka, C.A. Grande, Mater. Des. 187 (2020) 1.

[3] G.S. Falk, A.L. Rodriguez, W. Acchar, E.L. Foletto, Cerâmica 60, 353 (2014) 102.

[4] V. Shumilov, A. Kirilin, A. Tokarev, S. Boden, M. Schubert, U. Hampel, L. Hupa, T. Salmi, D.Y. Murzin, Catal. Today, in press.

[5] S.C. Daminabo, S. Goel, S.A. Grammatikos, H.Y. Nezhad, V.K. Thakur, Mater. Today Chem. 16 (2020) 1.

[6] M. Subramaniyan, S. Karuppan, P. Eswaran, A. Appusamy, A. Naveen Shankar, Mater. Today Proc. 45 (2021) 820 .

[7] C. Kukla, S. Cano, D. Kaylani, S. Schuschnigg, C. Holzer, J. Gonzalez-Gutierrez, Powder Metall. 62 (2019) 3. [8] L. Gorjan, C. Galusca, M. Sami, T. Sebastian, F. Clemens, Addit. Manuf. 36 (2020) 101391.

[9] A. Arnesano, K.P. Sanosh, A. Notarangelo, F. Montagna, A. Licciulli, Ceram. Int. 46 (2020) 2.

[10] S. Mamatha, P. Biswas, P. Ramavath, D. Das, R. Johnson, Ceram. Int. 44 (2018) 16.

[11] M.K. Agarwala, R. Van Weeren, A. Bandyopadhyayl, P.J. Whalen, A. Safari, S.C. Danforth, in Proc. Int. Solid Free. Fabr. Symp. (1996) 385.

[12] M.K. Agarwala, R. Van Weeren, A. Bandyopadhyay, A. Safari, S.C. Danforth, W.R. Priedeman, in Proc. Int. Solid Free. Fabr. Symp. (1996) 451.

[13] S. Azizi, C.M. Ouellet-Plamondon, P. Nguyen-Tri, M. Fréchette, E. David, Compos. B Eng. 177 (2019) 107288.

[14] H.F. Mark, Encyclopedia of polymer science and technology, $4^{\text {th }}$ ed., Wiley, New Jersey 6 (2014).

[15] K. Lin, R. Sheikh, S. Romanazzo, I. Roohani, Materials 12 (2019) 7.

[16] X. Wang, M. Jiang, Z. Zhou, J. Gou, D. Hui, Compos. B Eng. 110 (2017) 442.

[17] P. Parandoush, D. Lin, Compos. Struct. 182 (2017) 36. [18] I.J. Solomon, P. Sevvel, J. Gunasekaran, Mater. Today Proc. 37 (2021) 509.

[19] D. Nötzel, R. Eickhoff, T. Hanemann, Materials 11 (2018) 8.

[20] Z. Goharibajestani, O. Akhlaghi, C. Akaoglu, F. Afghah, 
N. Khani, A. Hodaei, B. Koc, O. Akbulut, ACS Appl. Polym. Mater. 1 (2019) 12.

[21] L. Poh, C. Della, S. Ying, C. Goh, Y. Li, Powder Technol. 328 (2018) 1.

[22] L. Gorjan, L. Reiff, A. Liersch, F. Clemens, Ceram. Int. 44 (2018) 13.

[23] D.W. Richerson, Modern ceramic engineering: properties, processing, and use in design, $3^{\text {rd }}$ ed., CRC Press, New York (2006).

[24] F. Thümmler, W. Thomma, Metall. Rev. 12 (2013) 69.

[25] J.E. Zorzi, C.A. Perottoni, J.A.H. da Jornada, Cerâmica 50, 315 (2004) 202.

[26] Reprap, reprap.org/wiki/RepRap/pt, ac. 07/01/2020.

[27] J. González-Gutiérrez, S. Cano, S. Schuschnigg, C. Kukla, J. Sapkota, C. Holzer, Materials 11 (2018) 1.

[28] I. Krindges, R. Andreola, C.A. Perottoni, J.E. Zorzi, Int. J. Appl. Ceram. Technol. 5 (2008) 3.

[29] R.A. Barbieri, C.A. Perottoni, J.E. Zorzi, Int. J. Appl. Ceram. Technol. 9 (2012) 3.

[30] R.H.R. de Castro, D. Gouvêa, Cerâmica 46, 300 (2000) 214.

[31] P.A. Ourique, R.C.D. Cruz, J.E. Zorzi, Cerâmica 61, 357 (2015) 71.

[32] M. Salehi, F. Clemens, E.H. Otal, D. Ferri, T. Graule, B. Grobéty, ChemSusChem 6 (2013) 2.

[33] M. Stumpf, N. Travitzky, P. Greil, T. Fey, J. Eur. Ceram.
Soc. 38 (2018) 10.

[34] E. Hnatkova, B. Hausnerova, P. Filip, Ceram. Int. 45 (2019) 16.

[35] W.N. dos Santos, Polím. Ciê. Tecnol. 15 (2005) 4.

[36] C. Rauwendaal, Int. AIP Conf. Proc. 1779 (2016) 30021.

[37] J.C. Wang, H. Dommati, S.J. Hsieh, Int. J. Adv. Manuf. Technol. 103 (2019) 2627.

[38] J. Webber, J.E. Zorzi, C.A. Perottoni, S. Moura e Silva, R.C.D. Cruz, J. Mater. Sci. 51 (2016) 5170.

[39] C. Kukla, J. Gonzalez-Gutierrez, I. Duretek, S. Schuschnigg, C. Holzer, AIP Conf. Proc. 190006 (2017) 5. [40] F. Bähr, E. Westkämper, Procedia CIRP 72 (2018) 1214. [41] P.K. Penumakala, J. Santo, A. Thomas, Compos. B Eng. 201 (2020) 108336.

[42] A.L.M. Carlos, "Utilização de tecnologia de impressão 3D para fabricação de arcabouços de PLA/hidroxiapatita carbonatada para regeneração óssea", Diss. Mestr., Un. Fed. Rio Janeiro, RJ (2016).

[43] N. Abbasi, H. Stephen, L.M. Robert, N.T. Nguyen, J. Sci. Adv. Mat. Dev. 5 (2020) 1.

[44] M. Janek, V. Zilinska, V. Kovar, Z. Hajduchova, K. Tomanova, P. Peciar, P. Veteska, T. Gabosova, R. Fialka, J. Feranc, L. Omanikova, R. Plavec, L. Baca, J. Eur. Ceram. Soc. 40 (2020) 4932.

(Rec. 21/05/2021, Rev. 19/07/2021, Ac. 23/07/2021) 